\title{
КЛИНИЧЕСКАЯ КИБЕРНЕТИКА В КОНТЕКСТЕ РАЗРАБОТКИ НОВЫХ ИНФОРМАЦИОННЫХ ТЕХНОЛОГИЙ НА ПРИМЕРЕ ТЕХНОЛОГИЙ АНАЛИЗА ЭЛЕКТРИЧЕСКОЙ АКТИВНОСТИ СЕРДЦА
}

\author{
И. А. Чайковский, И. Д. Войтович \\ Институт кибернетики им. В.М. Глушкова НАН Украины
}

This article focuses on clinical cybernetics as the basis of new information technologies development on the example of analysis of the heart electrical activity. A scheme of clinical information technology elaboration, which consists of 12 sequential steps is proposed, functions of medical specialist in clinical cybernetics is discussed.

Эффективная диагностика болезней сердца остается одной из главных задач современной медицины. Это обусловливается большой распространенностью и социально-экономической важностью таких заболеваний, прежде всего ишемической болезни сердца (ИБС), которая в последние десятилетия приняла характер пандемии. Особенно высок уровень заболеваемости и смертности от заболеваний сердца в Украине. Если в большинстве стран Европы на 1000 населения приходится не более 3 случаев смерти от сердечно-сосудистой патологии, то в Украине - более 9 случаев.

Понятно, что велика потребность в усовершенствовании методов диагностики заболеваний сердца, прежде всего неинвазивных методов, как наиболее доступных и безопасных. Анализ электрической активности сердца по прежнему является наиболее распространенным, доступным и дешевым методом объективного обследования сердца. Однако чувствительность и специфичность обычного электрокардиографического обследования недостаточно высоки. Известно, что ЭКГ покоя, оцениваемая по общепринятым критериям, остается нормальной приблизительно у 50 \% больных с хронической ишемической болезнью сердца, в том числе во время эпизодов дискомфорта в грудной клетке.

На протяжении многих лет в Институте кибернетики НАН Украины разрабатываются ряд современных информационных технологий на основе новых программно-аппаратных комплексов (ПАК), призванных увеличить эффективность анализа электрического генератора сердца. Среди этих комплексов следует отметить магнитокардиографические системы, системы ЭКГ 4-го поколения. Помимо этого разрабатываются и комплексы для оценки гемодинамики и микроциркуляции.

Клиническая кибернетика, как научная дисциплина, является основой создания этих современных средств функциональной диагностики и, в особенно- сти, имплементации их в практику лечебно-профилактических учреждений.

В этой связи надо заметить, что определение термина «клиническая кибернетика», как раздела медицинской кибернетики, не является устоявшимся.

Поскольку кибернетика - наука об общих законах управления и связи в организационных системах любой природы (машинах, живых организмах и обществе), то на наш взгляд, адекватным было бы следующее определение: клиническая кибернетика - это научная дисциплина, предметом которой является разработка и применение автоматизированных информационных систем и технологий, поддерживающих принятие всех возможных типов врачебных решений, а именно диагностических, прогностических, тактических (т.е. связанных с тактикой ведения больных в широком понимании этого слова), которые имеют место в клинической медицине.

Таким образом, центральным элементом дисциплины «клиническая кибернетика» являются клинические информационные технологии (ИТ), т.е. совокупность методов и программно-технологических средств, объединенных в технологическую цепочку, обеспечивающую сбор, хранение, предварительную обработку, интерпретацию, вывод и распространение информации. На выходе технологии образуется информационный продукт в форме, отвечающей потребностям конкретной предметной области, и с использованием «словаря» этой предметной области. В информационных технологиях, относящихся к клинической кибернетике, такой продукт - это автоматизированное диагностическое заключение, прогностический вывод или рекомендация по тактике ведения больного, в т.ч. медикаментозному или немедикаментозному лечению.

Как уже упоминалось, ИТ анализа электрического генератора сердца включают и разработку технических средств в виде программно-аппаратных комплексов.

(с) И. А. Чайковский, И. Д. Войтович 
Последовательность шагов (этапов) в рамках «технологической цепочки» современной информационной технологии анализа электрического генератора сердца представляется нам следующим образом.

Прежде всего, следует определить клиническую проблему, для решения которой создается ИТ. Понятно, что затрачивать интеллектуальные и материальные ресурсы для решения тривиальной задачи нецелесообразно. Поэтому ИТ создается для решения «трудных» клинических, прежде всего диагностических задач. Настоящая работа не ставит целью детальное обсуждение вопроса о том, что такое «трудная» диагностическая задача. Предложим здесь лишь неформальное определение такой задачи - это задача, для которой существующие диагностические алгоритмы имеют ограниченную точность, сложны или излишне трудоемки в реализации, или слишком дороги. Отсюда вытекает, что следующим шагом в развитии информационной технологии является прогнозная оценка ее реализуемости (feasibility) и экономических характеристик с точки зрения цена / эффект. Исходя из такой оценки составляется техническое задание для программноаппаратного комплекса, в котором описываются требования как к аппаратной, так и к программной части комплекса. Следующий этап заключается в реализации предложенного технического задания. Важно отметить, что в программном обеспечении, как правило, необходимо реализовать не только общепринятые, но и оригинальные показатели (биомаркеры), которые по замыслу их авторов обладают наибольшей информативностью в отношении решаемой задачи.

Следующим этапом развития ИТ является опытная эксплуатация нескольких разработанных экспериментальных образцов в лечебно-профилактических учреждениях. Затем наступает этап обратной связи, когда по результатам этой эксплуатации проводиться доработка разработанной технологии во всех аспектах, среди которых, кроме чисто технических, надо отметить структуризацию отображения и вывода информации для того, чтобы добиться наибольшей наглядности и удобства для пользователя. Затем следует этап информационной технологии, который можно назвать «аналитическим». Он подразумевает осмысление места разработанной технологии в имеющихся диагностических алгоритмах, ее «полезности» с точки зрения решения заявленной задачи, например уменышения неопределенности при принятии диагностического решения. Также имеет смысл повторно провести анализ технологии с точки зрения соотношения цена / эффект с учетом реальных затрат и достигнутой диагностической точности. Отдельно следует выделить этап формирования официальных методических рекомендаций по применению информационной технологии. Он знаменует собой принятие медицинским сообществом инновации, которую представляет собой разработанная информационная технология. Следующим этапом развития клинической ИТ является этап ее более или менее широкого использования в практике лечебно-профилактических учреждений. Конечно, этот этап также включает элемент обратной связи.

Если разработанная технология используется успешно и достаточно массово, она на следующем этапе может быть включена в национальные стандарты диагностики и лечения тех или иных заболеваний. Например, если речь идет о новых технологиях анализа электрической активности сердца, они могут стать частью стандартов диагностики тех или иных кардиологических заболеваний, прежде всего ИБС.

Наконец, завершающими этапами развития клинической информационной технологии, предназначенной для анализа электрического генератора сердца, могут стать международные многоцентровые исследования, опубликование метаанализов по результатам этих исследований, а затем положительная оценка новой информационной технологии авторитетными профессиональными организациями и/или страховыми компаниями, анализирующими новые клинические технологиям и определяющими политику в области клинической медицины (такими как NICE (Великобритания), Aetna (США) и другие), а также включение разработанной технологии в международные руководства по должной клинической практике (Good Clinical Practice - GCP).

Предлагаемая схема, состоящая из 12-ти последовательных шагов, конечно, не является исчерпывающей и неоспоримой. Однако, на наш взгляд, она удобна тем, что позволяет оценить степень зрелости любой клинической информационной технологии. Технологии, прошедшие этапы с первого по третий, должны быть классифицированы как находящиеся на начальной стадии разработки, с четвертого по восьмой - как находящиеся в промежуточной стадии, девятый или десятый этап - как зрелые, т.е. пригодные для практического использования и наконец, технологии, находящиеся двух заключительных этапах - как представляющие особую ценность. Примером зрелой клинической технологии, разрабо- 
танной в Институте кибернетики, является технология диагностики хронической ишемической болезни сердца с помощью магнитокардиографии.

В заключение целесообразно обсудить особенности функций врача - специалиста по клинической кибернетике. Этот специалист должен сочетать в себе профессиональные знания в области медицинской кибернетики и информатики с владением одной из клинических специальностей, в нашем случае - кардиологии или функциональной диагностики. Исходя из этого его естественная роль - быть инициатором создания клинической информационной технологии, выступать постановщиком задач на этапе формирования технического задания, предлагать оригинальные биомаркеры. Также он является ключевой фигурой на «аналитическом» этапе, этапе формирования методических рекомендаций. Иными словами, врач-кибернетик является неотьемлемой составной частью «коллективного конструктора» всегда имеющей междисциплинарную природу клинической ИТ. 